\title{
A frightening thought: Neuronal activity enhances tumor growth
}

Cell Research (2015) 25:891-892. doi:10.1038/cr.2015.65; published online 2 June 2015

Stem cells present in the adult brain are regulated by neuronal activity; malignant gliomas, which most likely originate from this population of cells, could also be regulated in this manner. A recent study by Venkatesh et al. published in Cell has identified Neuroligin-3 (NLGN3) as a mitogen promoting high-grade glioma growth.

Hardly any diagnosis is as terrifying to a patient as that of a primary brain tumor or glioma. In spite of major advances in our biological understanding, gliomas remain largely-untreatable often killing affected patients within 1-2 years of diagnosis. Among the most significant recent discoveries is that most, if not all, gliomas are likely to arise from stem cells that are present in the brain throughout life [1]. These include oligodendrocytes precursor cells (OPCs), which are destined to give rise to the cells that form the insulating myelin sheet around nerve fibers. OPCs are characterized by the expression of the transcription factor Olig-2 that represses the p53 tumor suppression pathway, allowing cells to continue dividing postnatally [2]. Consequently, these Olig-2-expressing cells have a heightened susceptibility to cancers as a result of somatic mutations in oncogenes.

OPCs have recently garnered significant attention in part because we now realize that they are involved in the myelination of nerve tracts well into early adulthood. This appears to be activity dependent and recent studies have shown that OPC proliferation is regulated by neuronal activity [3]. Given that these same cells could presumably give rise to gliomas, a new study by Venkatesh et al. [4] questioned whether neuronal activity may also influence the malignant growth of gliomas.

To tackle this question, the authors used a clever approach that allowed for the induction of neuronal activity on demand in a highly controlled fashion. This was achieved by introducing a light-activated cation channel, rhodopsin, into a select population of neurons in the premotor cortex. Simply shining blue light on the cortex leads to an influx of sodium ions via rhodopsin, thereby causing these neurons to fire action potentials. These transgenic mice were crossed into an immunodeficient background so that they could be used as recipients for human biopsy implants without tissue rejection. Implanted tumors grew at a steady rate with $\sim 7.5 \%$ of cells dividing at any given time. When neurons were photo-stimulated, however, the percentage of cells that divided increased by over $25 \%$. Importantly, when light activation was repeated daily for a week, the tumors were $42 \%$ larger than those in mice that lacked rhodopsin-expressing neurons, supporting the conclusion that neuronal activity somehow increases the proliferation of gliomas.

However, how might neurons control glioma cell proliferation? To answer this question, the team turned to a simpler slice culture preparation where they could sample the culture medium (CM) in which the slices where maintained. After photo-stimulation, CM was collected and applied to cultured glioma cells from 10 different patients. In 9 out of the 10 glioma cultures the medium caused a robust increase in cell proliferation. Next, the authors subjected the collected CM from stimulated slices to a series of biochemical analyses, including gel electrophoresis and mass spectrometry. This allowed for the identification of the factors responsible for the proliferation effect: neuroligin-3 (NLGN3), with a minor contribution from the two well-known growth factors brain-derived neurotrophic factor (BDNF) and glucose-regulated protein (GRP78).

NLGN3 is a cell-cell signaling protein primarily expressed on the postsynaptic terminal of excitatory synapses [5]. However, NLGN3 can also be cleaved and released, thereby acting as a diffusible signal. Its biological role beyond stabilizing synapses is not well understood, however, rare mutations in the $N L G N 3$ gene are found in patients with Autism spectrum disorder [6]. Interestingly, gliomas do not appear to harbor mutated NLGN3; hence the observed effects must be due to a change in the quantity of NLGN3 released, which was found to be elevated by 2.6 -fold in this study.

To demonstrate the necessity of NLGN3 for the increased proliferation observed, the authors showed that recombinant full-length human NLGN3 was sufficient to increase glioma proliferation. Furthermore, depletion of NLGN3 via its binding partner neurexin- $1 \beta$ reduced but did not completely abolish the growth-promoting effects. This reduction instead of com- 
plete abrogation is most likely due to the presence of the other two implicated mitogens in the CM, specifically BDNF. Finally, to gain an understanding of the intracellular signaling mechanisms, the authors employed RNA sequencing and pathway analysis, which converged on the PI3K-mTOR pathway. Inhibition of either PI3K or mTOR eliminated the NLGN3-mediated mitogenic effect.

Clearly, the principal strength of this paper is its detailed biological description unequivocally identifying NLGN3 as necessary and sufficient to mediate the growth-enhancing effect resulting from neural activity. The finding that neuronal activity promotes the growth of brain tumors using the same synaptic signaling molecules involved in normal synaptic development is entirely unexpected. However, the paper expands on a recent argument that gliomas must be examined from a more "neurocentric" perspective - in the context of the many interactions of the tumor with the surrounding brain tissue rather than merely a cancerous growth within the skull [7]. This realization is also supported by studies suggesting that glioma is a neurodegenerative disease $[8,9]$. Progressively exerting its detrimental function by disrupting the barrier function of endothelial cells, digesting the extracellular matrix, and releasing the neuronal excitatory neurotransmitter glutamate, causes seizures and neuronal cell death $[8,9]$. Understanding the molecular signals between all the involved cell types in the brain may allow us to gain a more holistic perspective of this devastating disease. This also holds the promise to find unexplored avenues for diagnosis, treatment, and possibly even prevention.

The application of new findings to the clinical management of patients is paramount. Many current experimental treatments are built on little biological understanding. Although bridging the findings in this study to the bedside will be challenging, this study still provides an important foundation for further investigation of neuronal control of glioma genesis. While we cannot silence neuronal activity to reduce the stimulating effect on the tumor, we can explore the use of drugs that may capture the released neuroligin, or interfere with the downstream activation of PI3K or
mTOR. The latter pathways are well established, with specific inhibitors in various stages of development.

\section{Emily G Thompson ${ }^{1}$, Harald Sontheimer $^{1}$}

${ }^{1}$ Department of Neurobiology, Center for Glial Biology in Medicine, University of Alabama at Birmingham, Birmingham, AL 35209, USA Correspondence: Harald Sontheimer E-mail: sontheimer@uab.edu

\section{References}

1 Liu C, Sage J, Miller M, et al. Cell 2011; 146:209-221.

2 Mehta S, Huillard E, Kesari S, et al. Cancer Cell 2011; 19:359-371.

3 Gibson E, Purger D, Mount C, et al. Science 2014; 344:1252304.

4 Venkatesh H, Johung T, Caretti V, et al. Cell 2015; 161:803-816.

5 Craig A, Kang Y. Curr Opin Neurobiol 2007; 17:43-52.

6 Tabuchi K, Blundell J, Etherton M, et al. Science 2007; 318:71-76.

7 Cuddapah V, Robel S, Watkins S, et al. Nat Rev Neurosci 2014; 15:455-465.

8 Watkins S, Robel S, Kimbrough IF, et al. Nat Commun 2014; 5:4196.

9 Buckingham SC, Campbell SL, Haas BR, et al. Nat Med 2011; 17:1269-1274. 